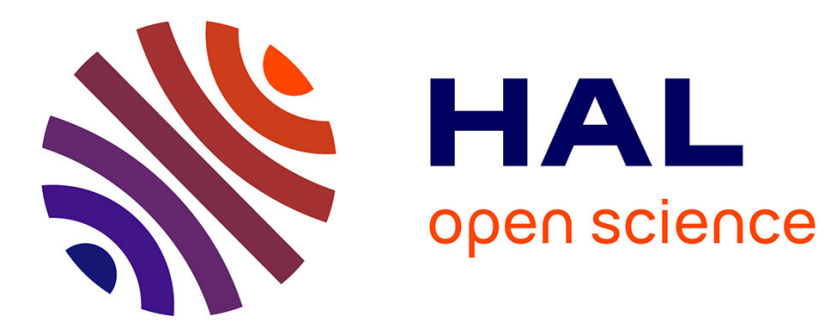

\title{
The Bethe lattice: a regular tiling of the hyperbolic plane
}

R. Mosseri, J.F. Sadoc

\section{To cite this version:}

R. Mosseri, J.F. Sadoc. The Bethe lattice: a regular tiling of the hyperbolic plane. Journal de Physique Lettres, 1982, 43 (8), pp.249-252. 10.1051/jphyslet:01982004308024900 . jpa-00232041

\section{HAL Id: jpa-00232041 https://hal.science/jpa-00232041}

Submitted on 1 Jan 1982

HAL is a multi-disciplinary open access archive for the deposit and dissemination of scientific research documents, whether they are published or not. The documents may come from teaching and research institutions in France or abroad, or from public or private research centers.
L'archive ouverte pluridisciplinaire HAL, est destinée au dépôt et à la diffusion de documents scientifiques de niveau recherche, publiés ou non, émanant des établissements d'enseignement et de recherche français ou étrangers, des laboratoires publics ou privés. 


\title{
LE JOURNAL DE PHYSIQUE-LETTRES
}

J. Physique - LETTRES 43 (1982) L-249 - L-252

15 AVRIL 1982, PAGE L-249

Classification

Physics Abstracts

$05.50-71.25 \mathrm{~L}$

\section{The Bethe lattice : a regular tiling of the hyperbolic plane}

\author{
R. Mosseri (*) and J. F. Sadoc $\left(^{* *}\right)$ \\ (*) Laboratoire de Physique des Solides, CNRS, 1, place A. Briand, 92190 Meudon, France \\ (**) Laboratoire de Physique des Solides (LA2), Université Paris-Sud, Bât. 510, \\ 91405 Orsay Cedex, France
}

(Reçu le 19 novembre 1981, accepté le 22 février 1982)

\begin{abstract}
Résumé. - On montre que le réseau de Bethe de coordinence quelconque peut être décrit comme un pavage régulier d'un plan hyperbolique. Un intérêt de cette description est de pouvoir construire des réseaux intermédiaires entre les réseaux réguliers du plan Euclidien et les réseaux de Bethe de même coordinence.
\end{abstract}

Abstract. - A Bethe lattice with a given coordination number can be described in terms of a regular
tiling of the hyperbolic plane. An interest of this description is the possibility of intermediate lattices
between regular lattices in Euclidean plane and Bethe lattices with the same coordination number.

1. Introduction. - The Bethe lattice has been widely used in solid state physics as it represents an underlying lattice for which many problems can be solved exactly [1]. It is often defined as a pseudo lattice since it cannot be embedded in any lattice belonging to an Euclidean space of finite dimension. The Bethe lattice is usually defined by the fact that it does not include any closed rings. An important property is that any vertex can be chosen as a reference vertex. This means that the first neighbour distances must be the same throughout the lattice. We intend to show in this paper that such a lattice can be described as a regular honeycomb in the hyperbolic plane. We first recall the conditions for the existence of these honeycombs.

We then present shortly the conformal model for the hyperbolic plane which is used in the figures. The Bethe lattice is shown to correspond to the special tilings denoted $\{\infty, q\}$.

2. Two dimensional honeycombs in the hyperbolic plane. - These tilings are denoted in a standard way using the Schlafli symbol $\{p, q\}$ where $p$ refers to the polygon ( $p=3$ for an equilateral triangle, $p=4$ for a square, etc...) and $q$ is the number of such regular polygons around each vertex. The tilings of the hyperbolic plane correspond to the integral values of $p$ and $q$ which satisfy the inequality [2] :

$$
(p-2)(q-2)>4 \text {. }
$$


This means that these tilings are infinite in number. On the contrary there are only a finite number of solutions for the spherical space in 2D (the 5 platonic polyhedra) and the Euclidean plane (the square $\{4,4\}$, triangular $\{3,6\}$ and honeycomb lattices $\{6,3\}$ ).

3. The conformal model. - The points of the hyperbolic plane are represented by points which belong to the interior of a fixed circle. Geodesic lines of the hyperbolic plane are now circles orthogonal to the fixed circle and reflections about these lines are replaced by inversion with respect to these circles.

A convenient method to build a given honeycomb in such a model is to generate all the vertices by successive reflections in the sides of the characteristic triangle associated with the polygon $p$, whose angles are $\pi / q, \pi / 2, \pi / p[2]$ (Fig. 1 ).

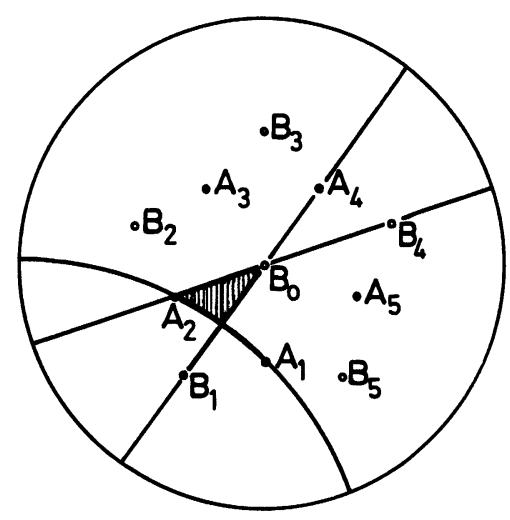

Fig. 1. - The characteristic triangle associated with the pentagon of the $\{5,4\}$ in the conformal model. The $A_{i}$ are vertices of the central pentagon. They are transformed into each other by inversion through the sides of the characteristic triangle as for the $B_{i}$ which are the centres of the pentagons (or the vertices of the dual tiling !).

Using this method one can build any of the $\{p, q\}$ where $p$ and $q$ satisfy equation (1). For example the $\{7,3\}$ and the $\{5,4\}$ are shown in figures $2 a$ and $b$. Due to the conformal representation the points are more and more gathered in the vicinity of the fixed circle. However the geodesic distance between two nearest neighbours is always the same throughout the honeycomb. This distance is simply related to the distance actually measured in this model (see Coxeter [2]). Any vertex close to the fixed circle perimeter can be chosen as the centre of new fixed circle using a suitably chosen isometry.

4. Curvature associated to the tessellation. $-\mathrm{A}\{p, q\}$ tiling with $p$-gon of unit edge length belongs to a surface of constant Gaussian curvature $K$. Which is related to the radius of curvature $K$ in spherical space by $K=1 / R^{2}$ and $K=-1 / R^{2}$ in the hyperbolic case. Let us call the edge length $2 \theta R . \theta$ is given [3] by :

$$
\cosh \theta=\frac{\cos \pi / p}{\sin \pi / q}
$$

which implies

$$
R=\frac{1}{2 \operatorname{argcosh}\left(\frac{\cos \pi / p}{\sin \pi / q}\right)}
$$


It is, for example, easily verified that $R$ is infinite for the regular tiling of the Euclidean plane $(K=0)$.

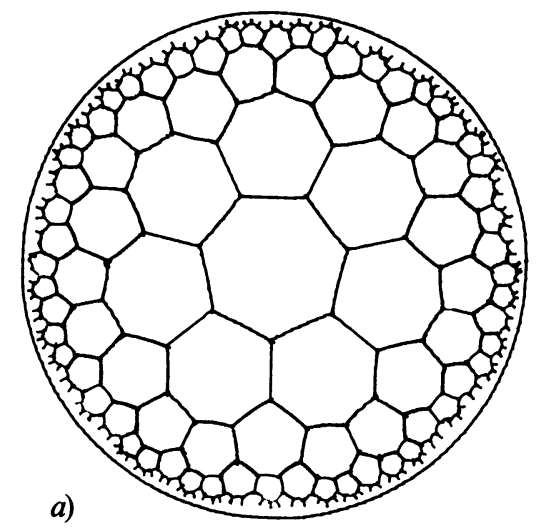

Fig. 2. - a) The honeycomb $\{7,3\}$ in the conformal model. b) The honeycomb $\{5,4\}$ in the conformal model.

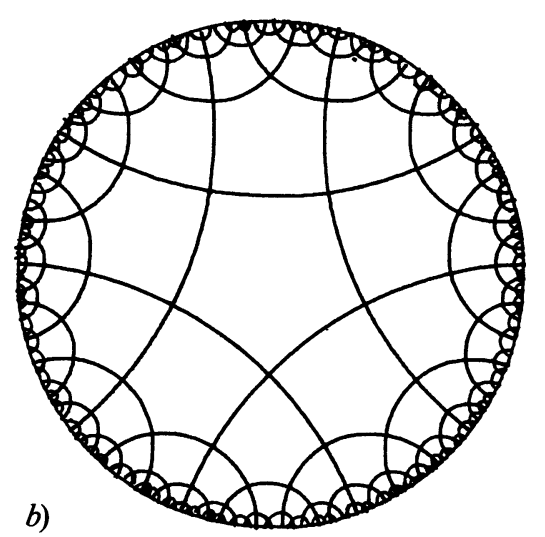

b)

5. The $\{\infty, q\}$ honeycombs. - In this picture it is possible to build, as a limiting case, a honeycomb $\{\infty, q\}$ whose cells are polygons with an infinite number of edges. The vertices of these polygons belong to circles of infinite length, different from straight lines (geodesics), which are called " horocyles " (see for example Toth [3]). The $\{\infty, 3\}$ is shown in figure 3. It is characterized by a constant vertex connectivity $(q=3)$, homogeneity (all vertices are equivalent) and the absence of any closed ring of finite length. The only difference with usual Bethe lattice is the presence of $q$ " $\infty$-gons " around each vertex. It is clear that these " $\infty$-gons " have no effect on any practical calculation. For example in the $« \mathrm{~s} »$ band tight-binding model, the moments $M_{n}$ of the density of states are related to $R_{n}$, the number of walks of $n$ steps in the lattice which start from a given vertex and return to this vertex. It is here evident that the $R_{n}$ of the usual Bethe lattice and of the $\{\infty, q\}$ are equal for every finite value of $n$, which means that the moments of the density of states are all equal.

It can be checked that the curvature associated with these honeycombs is well defined and increases with $q$.

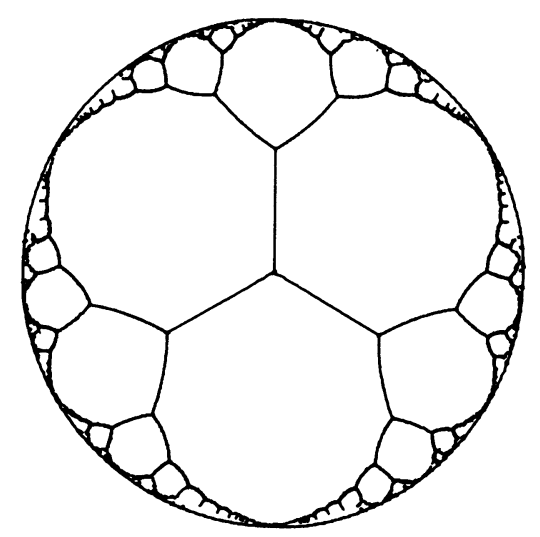

Fig. 3. - The honeycomb $\{\infty, 3\}$ in the conformal model. 
6. Conclusion. - We have shown in this paper that every Bethe lattice of coordination number $q$ can be described as a $\{\infty, q\}$, a regular tiling of the hyperbolic plane. A practical interest for this description comes from the remark that one can build an infinite and discrete set of lattices which lie in between the Bethe lattice and tilings of the Euclidean plane. When $q=4$ they are the $\{p, 4\}$ where $p$ runs from five to infinity, taking all the possible integer values. These lattices " connect " the square lattice $(\{4,4\})$ and the Bethe lattice of vertex coordination equal to 4 , the $\{\infty, 4\}$.

By joining the mid edges of the $\{p, q\}$ we obtain a quasi-regular tessellation denoted $\left\{\begin{array}{l}p \\ q\end{array}\right\}$ following Coxeter's notations [3]. In the case of the $\{\infty, q\}$ the new tessellation $\left\{\begin{array}{c}q \\ \infty\end{array}\right\}$ describes another family of pseudo-lattice : the Husimi cactus [1].

\section{References}

[1] THORPE, M. F., Bethe lattices in the NATO summer school on excitations in disordered systems, Michigan (1981). To be published.

[2] Coxeter, H. S. M., Non-Euclidean geometry (Univ. of Toronto Press).

[3] Coxeter, H. S. M. and Withrow, G. J., Proc. R. Soc. A201 (1950) 417.

[4] Tотн, F., Regular figures (Pergamon Press) 1964, p. 89, 97. 